\title{
Barrage de Marckolsheim
}

\section{Marckolsheim Dam}

\author{
PAR R. LESCAIL

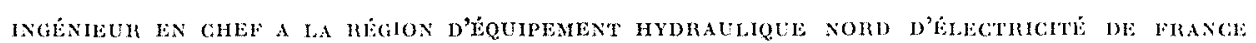

\begin{abstract}
Les quatre chutes abal de l'aménagement du Rhin entre Bale et Strasbonrg, comporteront chacune an barrage de retenue dans le fleube et sur la rive gauche, un canal de dérivalion alimentant lusine ef les ecluses de navigation. La premiere de ces quatres chutes, celle de Marckolsheim, est actuellement en cours d'exécution.

Le barrage devra permettre, en tenant compte des possibilités du canal de dérination, l'évacuation des crues polvant atteindre $6000 \mathrm{~m}^{\mathrm{s}} / \mathrm{s}$. Il comprendra cinq passes de $30,00 \mathrm{~m}$ de largeur sur $11,00 \mathrm{~m}$ de hateur, obturées par des vannes at deux corps independants. Chacun de ces corps sera manouvré par deax treails situés sur le cotronnement des piles.

Vne passerelle de service, située en amont des vannes, permettra la mise en place des éléments de batardeatux et l'acès aux différentes chambres de mécanismes.

Les travaux de constraction du barrage devront être exécutés sans interrompre la navigation.
\end{abstract}

\begin{abstract}
Each of the hydro-electric sites on the Rhine below Marckolsheim between Basle and Strasburg will each include a dam in the river, " diversion cand to supply the power house, and the locks required for navigation.

Marckholsheim, the first of these sites, is now at the construction stage.

The dam is designed to for a maximum flood discharge capacity of $6000 \mathrm{~m}^{3} / \mathrm{s}$, also allowing for the diversion canal's capacity. The dam will be provided wilh five stuices measuring $30 \mathrm{~m}$ wide $\times 11 \mathrm{~m}$ high, each of which will be filted with a split gate with two independently operating gate sections. Each of these sections will be controlled by twin hoists mounted on the pier top.

A service walkway will be provided upstream of the gates to enable the stop beams to be positioned, and as a means of access to the barious chambers housing the gate control gear. It is planned to carry out all the dam building worl without interrupting navigation on the river.
\end{abstract}

L'aménagement du Rhin entre Bàle et Strasbourg comportera au total huit chutes, dont les quatre chutes amont seront installées sur le Grand Canal d'Alsace et commandées par le seul barrage de Kembs, et dont les quatre chutes aval comprendront chacune un barrage de retenue installé sur le Rhin et une dérivation sur la rive gauche alimentant l'usine et faisant retour au Rhin en aval de l'usine. La navigation utilisera la retenue du barrage et le canal de dérivation qui comportera, au droit de l'usine, une écluse à deux sas, avec garages amont et aval.

Au total, cet aménagement comportera donc cinq barrages sur le fleuve.

\section{CARACTERISTIQUES GENERALES DES BARRAGES SUR LE RHIN}

Ces cinq ouvrages auront des caractéristiques générales très semblables, tant par leur hauteur voisine de $10 \mathrm{~m}$, que par la capacité d'évacualion des crues.
Cependant, si le barrage de Kembs a pu être construit sur une fondation rocheuse à faible profondeur, les quatre ouvrages restant à construire devront être fondés sur une masse d'allu- 
la retenue du barrage et qui, à l'aval, alimente les divers organes d'absorption du débit: groupes, déchargeurs, passes à glace, etc... de l'usine, aqueducs de remplissage et vidange des écluses, peut assurer l'évacuation d'une partie du débit de crue.

Pour conserver une marge de sécurité convenable, on a admis qu'on pourrait évacuer, en

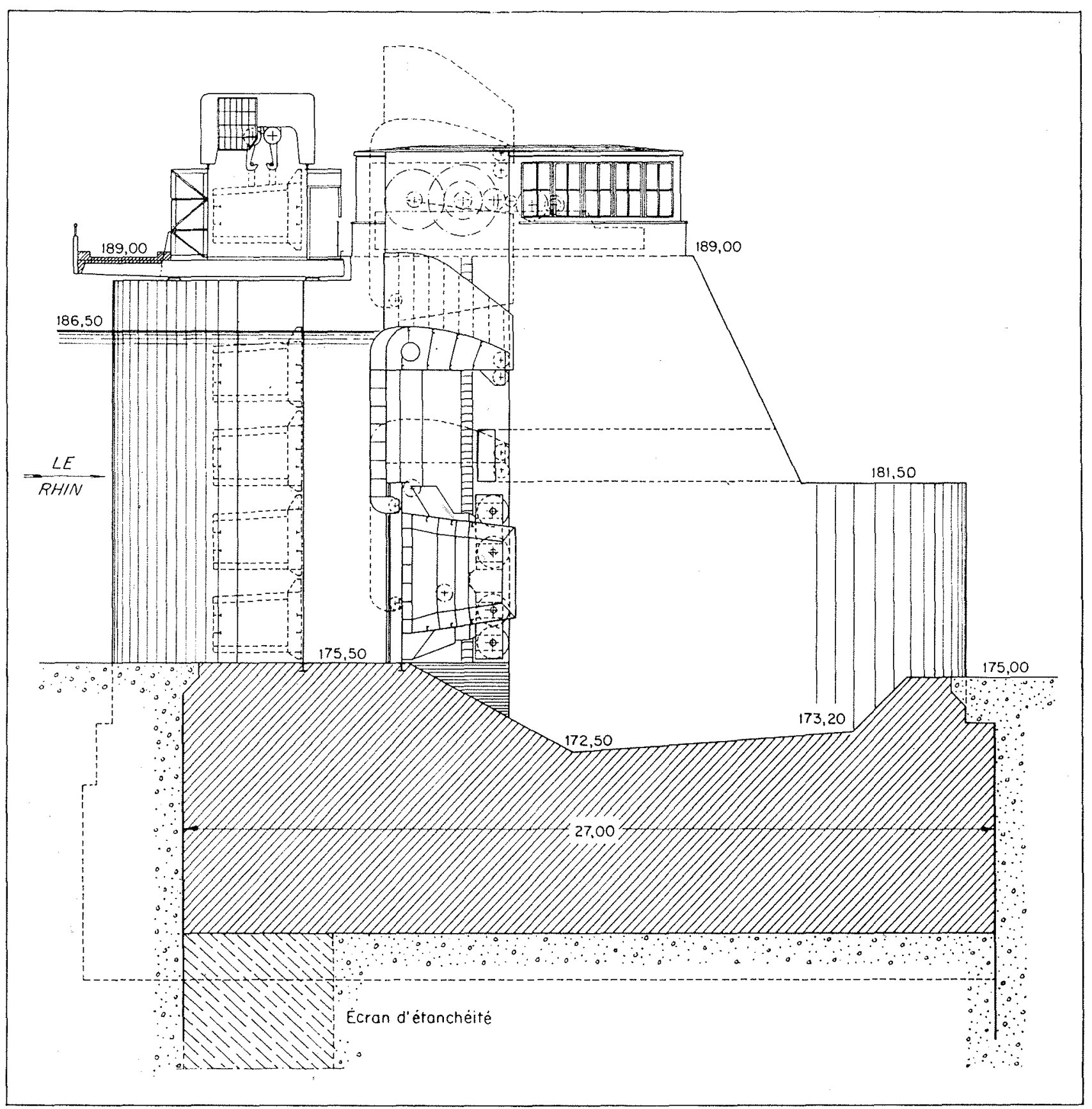

Fig. 1. Coupe transversale du barrage.

lout état de cause, par le canal, un débit égal à la moitié de la capacité totale des divers organes de l'usine et des écluses, soit $1200 \mathrm{~m}^{3} / \mathrm{s}$ compte tenu de la réduction de cette capacité due à la crue.
Le barrage seul doit donc être capable d'assurer l'évacuation d'un débit de $4800 \mathrm{~m}^{3} / \mathrm{s}$ et, toujours en vue de conserver une certaine marge de sécurité, il a été admis que ce débit devait pouvoir être évacué même si une des 
vannes du barrage était immobilisée, soit pour entretien, soit pour avarie.

Nous avons déjà signalé l'obligation d'assurer la passage de la navigation pendant les travaux. Cette sujétion liée à l'obligation d'exécuter à Marckolsheim, la plupart des piles situées dans le lit du fleuve avec fondations à l'air comprime - donc couteuses - nous a conduits à rechercher un ouvrage comportant un nombre réduit de piles avec des passes de grande largeur.

Ces diverses considérations nous ont amenés à retenir un ouvrage constitué par cinq passes de $30,00 \mathrm{~m}$ de largeur, obturées par des vannes levantes à deux corps indépendants, permettant le déversement de l'eau sur le corps supérieur de la vanne lorsqu'il est abaissé, et l'écoulement sous le corps inférieur lorsqu'il est levé. Les deux écoulements peuvent être réalisés simultanément au cours des manœuvres intermédiaires.

Les écoulements à travers une passe entièrement ouverte ont été déterminés par application des diverses formules usuelles et en particulier celle du professeur de Marchi, et ont été vérifiées sur modèle réduit au Laboratoire National d'Hydraulique de Chatou.

On a'adopté des vannes de $11,00 \mathrm{~m}$ de hauteur, bien que cette dimension ne soit pas nécessaire pour assurer l'évacuation de $1200 \mathrm{~m}^{3} / \mathrm{s}$ par passe. Cette disposition, avec seuil des vannes au niveau du fond $d u$ lit, doit permettre d'utiliser les passes dont les radiers sont déjà terminés, pour assurer le passage des eaux pendant les travaux, sans surélévation notable des niveaux:

Le débouché supplémentaire qu'offrent ces vannes pourra permettre si cela se révélait utile, d'effectuer au cours des crues, des abaissements de niveau de la retenue pour créer des chasses en vue d'entrainer les matériaux solides qui pourraient se déposer en amont du barrage.

Par ailleurs, en raison de l'obligation d'établir l'ouvrage sur un sol d'alluvions, le barrage a fait l'objet de nombreux essais sur modèle réduit au Laboratoire de Chatou pour étudier les risques d'affouillements.

En particulier, la forme et les dimensions à donner au radier onl été étudiées en vue de limiter les affouillements du lit en aval et surtout pour les reporter suffisamment loin en aval pour qu'ils soient sans danger pour la stabilité tes ouvrages.

Ces essais ont montré que les affouillements augmentaient rapidement dès que l'épaisseur de la lame déversante dépassait $3,00 \mathrm{~m}$. Il en a été tenu compte dans la conception des vannes.

Enfin, ces essais ont permis de préciser les protections qu'il y aura lieu de prendre pour éviter des affouillements en amont des piles.

\section{Caractéristiques particulières}

La coupe transversale du barrage donne les principales caractéristiques des diverses parties de l'ouvrage. (fig. 1)

Nous y ajouterons les quelques précisions suivantes:

\section{a) VANnes :}

Les vannes sont du type à deux corps indépendants.

Le corps inférieur, en forme de caisson fermé, s'appuie sur ses chemins de roulement par huit galets de $1,00 \mathrm{~m}$ de diamètre montés sur boggies.

Le corps supéricur, dont la course est limitéc à $3,00 \mathrm{~m}$, s'appuie sur ses chemins de roulement latéraux par quatre galets de $500 \mathrm{~mm}$. et sur le corps inférieur par douze galets de $350 \mathrm{~mm}$. Il comporle à sa partie supérieure une poutre de rigidité formant guidage de la lame déversante.

Les treuils de manouvre, au nombre de deux pour chaque corps de vanne, sont logés dans des chambres situées sur le couronnement des piles. Ils sont synchronisés électriquement.

Les vitesses de manœurre des vannes sont de: $0,20 \mathrm{~m}$ par minute pour la vanne inférieure,

$0,40 \mathrm{~m}$ par minute pour la vanne supérieure.

Chaque vanne sera commandée localement à partir de la chambre de mécanisme voisine.

Deux des vannes centrales sont prévues pour être équipées avec commande automatique.

\section{b) Batardeau amont :}

Il comportera quatre éléments de même hauteur correspondant à l'obturation d'une passe.

Ces éléments seront stockés, au droit des passes, au-dessus du couronnement des piles, entre les deux poutres de la passerelle de service.

Un engin de manceuve de ces éléments roulera sur les deux poutres du pont de service, situées de part et d'autre de la rainure des batardeaux. Il comportera deux chariots espacés de $30,00 \mathrm{~m}$ et reliés rigidement entre eux. Chacun de ces chariots comporte un treuil avec dispositif d'accrochage des éléments de batarteau, guidé dans les rainures. Les deux treuils sont synchronisés par un arbre.

L'engin permettra de déposer un élément sux le terre-plein de rive gauche, en vue d'une réparation.

La vitesse de levage est de $3,00 \mathrm{~m}$ par minute.

\section{c) Circulation de service:}

Le pont de service comporte deux poutres situées de part et d'autre de la rainure des batardeaux et servant de chemin de roulement à l'engin de manœuvre. 
La poutre amont porte un tablier de 2,50 $\mathrm{m}$ de largeur, qui permet, au droit de chaque pile, l'accès aux chambres de mécanismes. La poutre aval comporte une passerelle abritée pour le personnel, qui relie les diverses chambres de mécanismes entre elles. Elle sert en outre au passage des câbles.

\section{Importance des ouvrages}

La construction du barrage de Marckolsheim entraînera la mise en œuvre de :

$65000 \mathrm{~m}^{3}$ de bétons divers, et de $700 \mathrm{t}$ d'aciers d'armature.

En outre, l'équipement mécanique des vannes, batardeaux et organes de manœuvre représentera un tomnage total d'environ $2500 \mathrm{t}$.

\section{EXECUTION DES TRAVAUX}

\section{a) Programme :}

Le programme de construction du barrage de Marckolsheim a été établi en vue de ne pas apporter de gêne à la navigation dont nous avons signalé l'importance.

Il était pour cela indiqué, pendant toute la durée des travaux pendant laquelle les bateaux doivent traverser le chantier, de conserver au chenal de navigation, sa largeur et, autant que possible, son emplacement actuel. Un déplacement notable de ce chenal ne pourrait en effet être réalisé, qu'en modifiant le système des épis sur plusieurs centaines de mètres à l'amont et à l'aval du chantier. Une telle modification ne serait pas sans provoquer de gêne pour la circulation des bateaux; elle risquerait en outre de perturber l'équilibre actuel du lit.

D'autre part, il était nécessarre, malgré l'encombrement progressif du lit dû à la construction des ouvrages de ne pas augmenter les vitesses d'écoulement dans le chenal et surtout de ne pas y provoquer d'augmentation locale de la pente superficielle, susceptible de majorer considérablement les puissances nécessaires à la remontée des bateaux en hautes eaux. liti, 2.

Phases de construction.

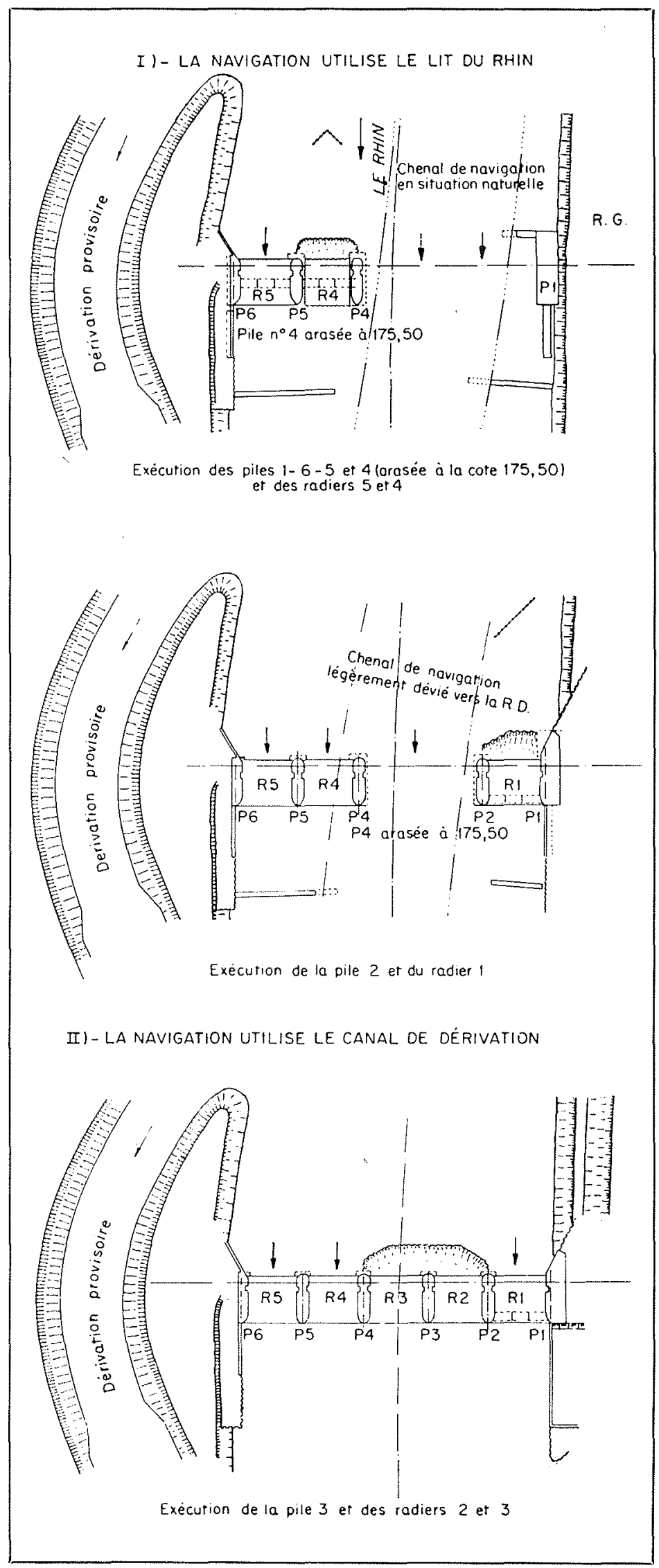




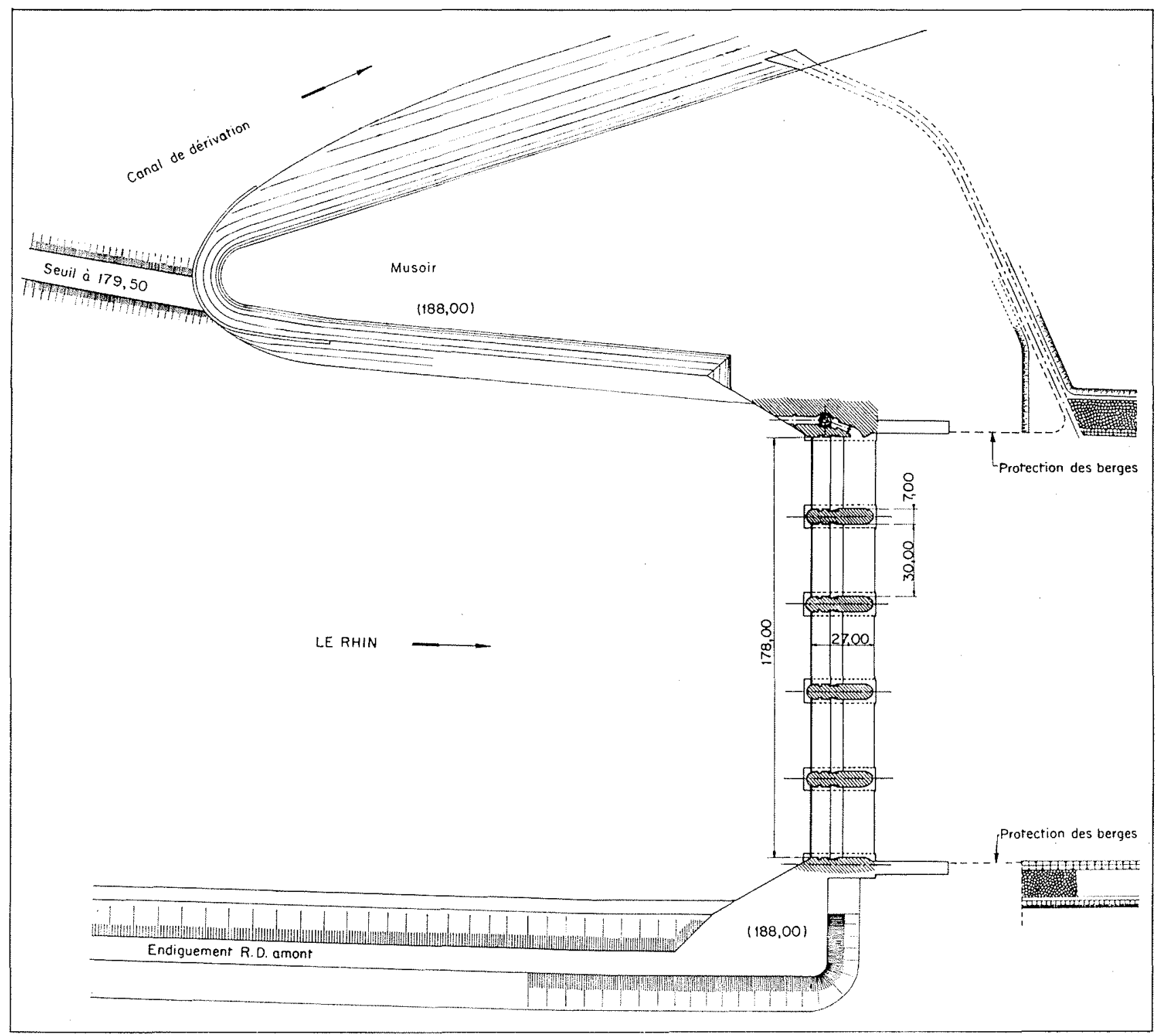

fiki. 3. Plan général.

Cette question a été longuement étudiée sur modèle réduit, pour les phases les plus délicates.

Ces dernières conditions ont pu être entièrement satisfaites par la création, sur la rive droite, d'une courte dérivation provisoire qui compense la réduction de section due aux ouvrages en assurant le passage d'environ 15 à $20 \%$ du débit du fleuve.

Grâce à cette disposition, les vitesses dans le chenal navigable maintenu dans le lit du fleuve restent inférieures ou au plus égales aux vitesses actuellement observées, quel que soit le débit.

En basses eaux, la présence de la dérivation provisoire ne modific pas la profondeur d'eau dans le chenal.
La figure 2 donne le schéma des principales phases de construction,

b) Procḱdés de construction :

Seules les trois piles en rivièe n" 2, 4 et 5 seront exécutées à l'air comprimé.

Tous les autres ouvrages: piles-culées, radiers et pile $n^{\circ} 3$ seront exécutés à sec à l'intérieur. d'enceintes en palplanches métalliques, après étanchement du fond de ces enceintes par une couche convenable de béton coulé sous l'eau.

Ces enceintes prendront appui sur les piles déjà exécutées à l’air comprimé.

L'exécution de ces enceintes et en particulier les opérations de coupure du courant, ont fait l'objet d'études sur modèle réduit. 